\title{
PERSPECTIVA TEMPORAL FUTURA Y METAS DE VIDA EN ADOLESCENTES ESTUDIANTES DE NIVEL MEDIO
}

\author{
Mariana Jaime \\ Universidad Abierta Interamericana \\ Facultad de Psicología y Relaciones Humanas \\ marianaandreajaime@gmail.com \\ Carolina De Grandis \\ Universidad Abierta Interamericana \\ Facultad de Psicología y Relaciones Humanas \\ Instituto de Investigaciones, Facultad de Psicología \\ Universidad de Buenos Aires. Laboratorio de Cognición y Políticas Públicas \\ Consejo Nacional de Investigaciones Científicas y Técnicas (CONICET) \\ Lucas G. Gago-Galvagno \\ Universidad Abierta Interamericana \\ Facultad de Psicología y Relaciones Humanas \\ Instituto de Investigaciones, Facultad de Psicología \\ Universidad de Buenos Aires. Laboratorio de Cognición y Políticas Públicas \\ Consejo Nacional de Investigaciones Científicas y Técnicas (CONICET)
}

Recepción Artículo: 16 septiembre 2020

Admisión Evaluación: 23 octubre 2020

Informe Evaluador 1: 25 noviembre 2020

Informe Evaluador 2: 23 noviembre 2020

Aprobación Publicación: 27 noviembre 2020

\section{RESUMEN}

En la presente investigación se examinarán las relaciones entre la Perspectiva temporal futura y las Metas de vida en adolescentes estudiantes de nivel medio. Se trabajó con 110 estudiantes de escuelas secundarias de la Provincia de Buenos Aires. Se utilizó para la recolección de datos una encuesta de datos sociodemográficos, la adaptación local del inventario de Perspectiva Temporal de Zimbardo realizada por Galarraga y Stover (2016) y la escala de Metas de Vida de Cattaneo \& Schmidt (2014). Los resultados indican que las personas que se orientan al presente hedonista disfrutan del momento presente, evitan responsabilidades y presentan aspiraciones de tipo solidarias, entre otras metas. En la actitud negativa hacia el presente los adolescentes tienden a conformarse, pero esto no sucedería con aquellos que se orientan negativamente hacia el pasado. Además, en ambas orientaciones temporales no se evidencian metas que impliquen una planificación real hacia el futuro. En la orientación al futuro capacitarse para los adolescentes no constituirá un medio para lograr metas. Con relación a la edad se hallaron diferencias significativas, los de menor edad presentan metas afectivas y se orientaron al presente hedonista y fatalista. Los futuros egresados en electromecánica a diferencia de los futuros maestros mayores de obra estiman 


\section{PERSPECTIVA TEMPORAL FUTURA Y METAS DE VIDA EN ADOLESCENTES ESTUDIANTES DE NIVEL MEDIO}

obtener un trabajo bien remunerado a partir del título obtenido. Se concluye que las metas establecidas por los adolescentes varían en función de la orientación temporal que prevalezca sobre ellos.

Palabras clave: perspectiva temporal futura; metas de vida; adolescencia

\section{ABSTRACT}

Future time perspective and life goals in adolescent students of medium level. In the current investigation we are going to examine the relationships between the Future temporal perspective and the Life goals in adolescent students in high school. The study was conducted with 110 students from high schools from the province of Buenos Aires. In order to collect data, we carried out a sociodemographic survey, as well as using the local adaptation of Zimbardo Time Perspective inventory by Galarraga and Stover (2016) and the Life goals scale by Cattaneo \& Schmidt (2014). The results show that the people with a present hedonist orientation enjoy the present, avoid responsibilities and have solidary aspirations. In a negative attitude towards the present, adolescents tend to conform, while this would not be the case with those with a negative attitude towards the past. Besides, there is no evidence of goals in relation to the future in both time orientations. In the future orientation, training is not a means to achieve goals for adolescents. As far as ages are concerned, we found significant differences, with the youngest showing affective goals and a present and fatalistic hedonist orientation. The future electro mechanic graduates unlike the future master builders' graduates expect to get a well-paid job as a result of their degree. We conclude that the goals set by adolescents vary according to the time orientation that prevails over them.

Keywords: temporal perspective; life goals; adolescence

\section{INTRODUCCIÓN}

Lilo Espinoza (2004) indica que la adolescencia constituye un período especial del desarrollo, del crecimiento y de la vida de cada individuo debido a que no solo es una fase de transición entre el estadio infantil y el adulto, sino que se trata de una etapa de constitución de la identidad definitiva de cada sujeto, la cual conlleva un trabajo mental gradual, lento y lleno de dificultades. A su vez, Cattaneo \& Schmidt, (2014) entienden la adolescencia como una etapa decisiva del desarrollo que apunta a una mayor independencia psicológica y Carcelén Velarde (2008), considera que los cambios que se producen en la misma afectan los diversos ámbitos en los que los adolescentes se comportan, marcando su incorporación a la vida adulta, la cual se caracteriza por la formación superior, capacitación para el empleo, la independencia y la intimidad.

Con relación a la percepción subjetiva del tiempo, la misma viene siendo estudiada por los psicólogos desde hace más de un siglo ya que consideran que constituye uno de los elementos principales de la cognición, debido a que les da sentido a las experiencias vividas (James, 1890 citado en Del Rio \& Herrera, 2006), permite autorregular el comportamiento presente (Lewin, 1935) y anticipar el futuro (Fraisse, 1983).

Zimbardo \& Boyd (1999), definen la perspectiva temporal futura (en adelante PTF) en términos de orientación temporal, que alude a la tendencia del sujeto a estar centrado en el pasado, presente o futuro. Este es un proceso no consciente, que ocurre en el sujeto sobre el cual organizaría sus vivencias personales y sociales en categorías temporales, las cuales contribuyen a la codificación, almacenamiento y evocación de situaciones que han sido vivenciadas, así como también ejercen un rol primordial en la construcción de metas y expectativas a futuro. La orientación en el tiempo de la que disponen las personas tiende a prevalecer una sobre la otra otorgando sentido y significación al comportamiento e influyendo en la toma de decisiones (Zimbardo, 1999). Los autores sostienen que la perspectiva temporal se asocia a la forma de actuar, a las creencias, a las valoraciones que se realizan sobre Ios acontecimientos y a las experiencias de las personas, las cuales constituyen un factor de importante impacto en el comportamiento. Por lo tanto, la valoración del tiempo es relevante para la vida diaria de las personas, aunque no se sea consciente de ello.

Zimbardo y Boyd (1999) desarrollaron cinco dimensiones temporales, vinculadas a tres núcleos temporales pasado, presente y futuro. La primera dimensión hace referencia al pasado positivo, y se relaciona con las viven- 
cias de las personas que resultaron agradables y placenteras. La segunda dimensión pasado negativo expresa una visión negativa y de rechazo hacia las experiencias del pasado, que se vinculan con sucesos que poseen una carga negativa caracterizados por resultar difíciles y complicados. El presente hedonista constituye la tercera dimensión y se relaciona con la búsqueda de sensaciones placenteras en las situaciones que se viven diariamente, con muy poca consideración de las consecuencias de estas para el futuro. Se asocia a vivir el momento presente, a obtener placer espontáneo y a obtener gratificación inmediata. La cuarta dimensión, presente fatalista se asocia a las experiencias actuales del sujeto que resultan generadoras de ansiedad y temor. Por último, la quinta dimensión hace referencia al futuro asociado a la planificación de acciones en concordancia con las metas que las personas se proponen lograr en la vida.

Las metas son entendidas por Austin y Vancuver (1996) como representaciones internas de los estados deseados, las cuales constituyen la representación cognitiva de qué es lo que un sujeto está intentando alcanzar en una situación determinada (Ford, 1992; Wentzel, 1992,1999). Por su parte, Cattaneo \& Schmidt (2014) consideran que las metas se elaboran en términos de una dialéctica psico-histórica, es decir en una interacción constante entre la historia personal, las vivencias actuales y el horizonte de expectativas. Es a partir de estos tres dominios pasado, presente o futuro que el sujeto puede elaborar una meta desde la cual construirá su propia identidad. Asimismo, para la construcción de metas de vida es necesario una serie de andamiajes fundamentales para que las mismas puedan llevarse a cabo con éxito como ser; la identidad, los intereses, el autoconcepto y las disposiciones básicas de cada sujeto, como también las oportunidades que el medio provee, el acceso a los bienes y servicios, la calidad de vida y el entorno ecológico. Por lo tanto, son múltiples los condicionantes que le proporcionan al sujeto un espacio donde se gestan, se construyen proyectos y se toman decisiones.

Las autoras han categorizado diez metas de vida asociadas a dominios relevantes emparejados con el período de vida adolescente: Moral Social Familiar; el sujeto considera la importancia de proyectarse hacia el encuentro exogámico, considerando valores personales y propios de la cultura familiar. Afectivo Interpersonal; hace referencia a las motivaciones intrínsecas de orden psicoafectivo del sujeto relacionadas a la autopercepción de inestabilidad emocional, conflictos y confrontación con las figuras parentales. Logros Materiales y Prestigio; reúne ideas competitivas y de ambición personal, destacando una motivación para lograr sobresalir y ejercer liderazgo. Logro Económico-Laboral; acopia expectativas de logro económico ya sea a través del estudio o de un título que le permitan alcanzar un trabajo remunerado. Afiliativa; son metas relacionados a la "deuda generacional" es decir, se sostienen en ideas de retribución al esfuerzo realizado por los padres. Idealista-Altruista; hace referencia a la coexistencia de ideas y pensamiento constituidos por la voluntad del individuo en función de metas desiderativas. Presión Contextual; manifiesta las presiones o mandatos que el contexto familiar y social ejerce sobre el sujeto en el momento de desarrollar un proyecto personal. Capacitación como medio; se relaciona con la búsqueda de metas vinculadas a la capacidad educativa como un medio, como un "saber hacer algo" más que como un fin en sí mismo. Hedonista; hace referencia a la tendencia de los adolescentes a realizar sólo aquello que tienen ganas, evitando dificultades y posponiendo responsabilidades el mayor tiempo posible. EstándarConformista; se relaciona con el modo en que el sujeto se piensa cuando se encuentra frente a una posibilidad laboral marcada muchas veces por la aceptación resignada y conformista ante las dificultades que plantea el entorno.

Se han desarrollado estudios que analizan la relación entre la PTF y las metas de vida, uno de ellos es la investigación llevada a cabo en Argentina por de Difabio de Anglat, Vásquez y Noriega Biggio (2018) quienes verificaron que la orientación al presente y pasado negativista se relaciona con metas referidas al self, a los logros académicos y laborales y a los valores sensibles tales como las posesiones materiales y a las diversiones. En cambio, la orientación positiva identificó que las personas mencionan metas referidas al contacto social (altruista e íntimo), a la autorrealización (profesional y de carácter religiosa) y a los valores del sentido de la vida. Con respecto a la edad, el grupo de adolescentes muestra valores más altos en presente hedonista y fatalista y los más bajos en futuro. Con relación a las metas se halló una única diferencia en la meta de contacto social (altruista) con nivel más alto para las mujeres. Fernández \& Macbeth (2018) también en Argentina realizaron un estudio sobre la perspectiva temporal futura, metas y submetas y su rol en la toma de decisiones y hallaron que los pun- 
tajes altos en perspectiva de futuro se inclinan más al logro de la meta y las sub-metas facilitan la toma de decisiones. A su vez, Pérez, Guilianu \& Soliverez (2014) realizaron en la ciudad de Mar del Plata (Argentina) un estudio orientado a explorar la posible relación entre las metas vitales, la PTF y los vínculos positivos en hombres y mujeres, adultos jóvenes y adultos mayores. Los resultados evidenciaron que los adultos jóvenes priorizan el establecimiento de vínculos menos íntimos, pero con personas que consideran interesantes, en tanto los mayores prefieren los vínculos más cercanos. Además, los más jóvenes se plantean metas a futuro vinculadas con el logro de ciertos roles o a la adquisición de estatus en el ambito familiar, laboral y/o formación. En cambio, Ios mayores mencionan metas a corto plazo, vinculadas al presente y focalizadas en la salud y en mantener una situación de bienestar óptima.

Las investigaciones realizadas sobre la PTF coinciden en que la orientación al Futuro se asocia a aspectos positivos como, por ejemplo: mejor planificación, mejor rendimiento académico, logros laborales, conductas saludables entre otras (Lens, Paixaó, Herrera \& Grobler, 2012).

Un estudio realizado por Dauda \& Durand (2018) encontró asociaciones entre el compromiso académico y la orientación temporal en estudiantes argentinos. Se halló que el valor brindado al aprendizaje de nuevos contenidos y el interés por aprenderlos se asocian a una perspectiva temporal equilibrada y la capacidad de regular el espacio y la agenda personal estaría en vinculación con la visión que se tiene del Futuro.

Galarraga y Stover (2017) estudiaron la perspectiva temporal y las estrategias de afrontamiento en estudiantes de nivel medio próximos a egresar hallando una vinculación entre el pasado negativo y la evitación conductual, entre el presente hedonista y las estrategias de aproximación cognitiva y evitación conductual. Con relación a la PTF y al curso se halló que cuanto más jóvenes resultan los sujetos, mayor es la tendencia al presente fatalista. En México, Robles Ojeda, Galicia Moyeda \& Sánchez Velasco (2017) realizaron una investigación que tenía como objetivo identificar el perfil en orientación temporal, autorregulación y aproximación al aprendizaje hallando que los puntajes altos en la orientación al futuro y bajos en la orientación al pasado negativo se asocian a un mayor rendimiento escolar, uso de estrategias de autorregulación y una aproximación profunda al aprendizaje.Además, un estudio realizado por Vásquez, Difabio de Anglat \& Noriega (2016) que exploró la relación entre la perspectiva temporal y los estilos de personalidad en estudiantes argentinos, halló que los estudiantes que se orientan al futuro poseen un compromiso académico profundo y un estilo de personalidad motivacionalmente dirigido a proteger y resguardar las necesidades ajenas; cognitivamente propensos a la sensación y sistematización, en los vínculos se muestran más comunicativos, solidarios y capaces de crear un buen vínculo laboral.

En los últimos años se han realizado diversos estudios acerca de la relación entre la PTF y las metas de vida, pero hasta el momento no se ha utilizado la Escala de Metas de vida desarrollada por Cattaneo \& Schmidt (2014) empleada en el presente trabajo, por lo tanto, este estudio permitiría obtener nueva información sobre esta relación que posibilite ampliar las teorizaciones y otorgar evidencia empírica. A su vez, los resultados de la presente investigación permitirán tener una visión más clara de la relación entre las variables y de esta manera contribuir al desarrollo de un proceso de orientación académico que acompañe a la elaboración del proyecto personal de los adolescentes.

El presente trabajo tiene como objetivo analizar las posibles asociaciones entre las dimensiones de la PTF y las dimensiones de las metas de vida en adolescentes estudiantes de nivel medio. Se espera encontrar que la orientación al presente hedonista correlacione con las metas hedonistas y altruistas, que el presente y pasado negativo se asocie a las metas estándar-conformista y de presión contextual, que el futuro correlacione con las metas logros materiales y prestigio, logro económico- laboral, idealista-altruista y capacitación como medio. A su vez, no se espera encontrar diferencias en las metas según la edad, se espera encontrar diferencias en la PTF en relación con la edad. También se espera encontrar que los adolescentes con orientación técnica presenten metas de Logro Económico-Laboral. 


\section{MÉTODO}

\section{Diseño}

El presente trabajo es una investigación de tipo cuantitativa, no experimental, transversal y con alcance correlacional y comparativo.

\section{Participantes}

Se evaluaron 110 adolescentes (femenino=39) con distintas orientaciones educativas (electromecánica, construcciones, ciencias naturales y economía y administración) de entre 15 y 19 años ( $M=16.53, D S=0.72$ ), pertenecientes a la localidad y partido de Lomas de Zamora.

Se utilizó un tipo de muestreo no probabilístico por conveniencia. Los criterios de inclusión fueron estudiantes que estuvieran cursando los últimos años de la escuela secundaria, excluyendo a quienes omitan preguntas en alguna de las pruebas o que solo completen un cuestionario.

\section{Instrumentos}

Cuestionario de datos socio demográfico. Recoge información sobre la edad, identidad de género, lugar de residencia, grupo familiar con el que convive, nivel de estudio de los padres, si los padres trabajan o no, orientación educativa y año en curso.

Escala de Metas de vida para adolescente (EMVA) de Cattaneo y Schmidt (2014). La misma consta de 87 ítems distribuidos en 10 subescalas. Cada ítem posee una escala tipo Likert con cinco opciones de respuesta (desde totalmente en desacuerdo hasta totalmente de acuerdo). El Alpha de Cronbach en las subescalas presentó niveles que oscilan entre 0.56 y 0.85 . Además, presenta validez aparente (face validity), ya que la valoración que Ios usuarios realizan de la prueba permite afirmar que mide su constructo meta. El coeficiente Alpha de Cronbach de la presente investigación es de $=0.662$. Los coeficientes Alpha de Cronbach de las dimensiones Metas de Vida son: $=0.629$ para Moral-Social-Familiar, $=0.630$ para Afectivo-Interpersonal, $=0.638$ para Logros Materiales y Prestigio, $=0.629$ para Logro Económico y Laboral, $=0.630$ para Afiliativa, $=0.631$ para IdealistaAltruista, $=0.654$ para Presión Contextual, $=0.650$ para Capacitación como Medio, $=0.646$ para Hedonistas, $=0.647$ para Estándar-Conformista.

Inventario de Perspectiva Temporal Futura (Zimbardo y Boyd, 1999), el cual fue traducido, validado y adaptado para la población local adolescente estudiantil por Galarraga y Stover (2017). Consta de 34 ítems organizados en cuatro factores, pasado negativo, presente hedonista, presente fatalista y futuro. Los elementos poseen un formato de respuesta Likert de 4 posiciones que van desde nada hasta mucho. El Alpha de Cronbach en las subescalas presentó niveles que oscilan entre 0.80 y 0.59. Además, se realizaron estudios de evidencia de validez de criterio seleccionando como criterio externo diversa información académica en tres momentos temporales: repitencia (pasado), asignaturas adeudadas (presente), y la elaboración de proyectos tras el egreso (futuro). La confiabilidad Alpha de Cronbach del presente trabajo son $=0.601$ para el Presente Hedonista, $=0.699$ para el Presente Fatalista, $=0.684$ para el Pasado Negativo y $=0.644$ para el Futuro.

\section{Procedimiento}

Se contactó a estudiantes que concurren a un instituto tecnológico y a una escuela de nivel medio, ambos de la localidad y partido de Lomas de Zamora. Se les brindó una explicación general sobre la finalidad de la investigación para luego proceder a la administración en forma colectiva de los instrumentos y del cuestionario de datos sociodemográficos. Las pruebas fueron realizadas de forma anónima y voluntaria. Administrándose bajo el consentimiento previo de los alumnos, de la escuela y de los padres de los integrantes de la misma.

Los datos recopilados fueron procesados a través del software IBM-SPSS (Statistical Package for the Social Sciences), versión 22. En el caso de la variable edad, la misma fue recodificada, quedando la misma conformada 
en dos rangos: adolescentes de 16 años o menos y adolescentes de 17 años 0 más. Esto se realizó considerando la investigación realizada por Galarraga y Stover (2017).

\section{RESULTADOS}

Se realizó la prueba de normalidad de Kolmogórov-Smirnov para indagar la distribución de las variables. La mayoría de las variables se distribuyeron de forma anormal $(p<0.05)$. Por lo tanto, se determinó utilizar estadísticos no paramétricos para realizar los análisis posteriores.

\section{Correlación de variables}

Se aplicó una prueba de correlación Rho de Spearman para probar las hipótesis de asociación de variables. El resumen de los resultados se encuentra en la Tabla 1.

Tabla 1. Correlación entre las dimensiones de la variable Perspectiva Temporal Futura y Metas de Vida

\begin{tabular}{|c|c|c|c|c|c|}
\hline & & $\begin{array}{l}\text { Presente } \\
\text { Hedonista }\end{array}$ & $\begin{array}{l}\text { Presente } \\
\text { Fatalista }\end{array}$ & $\begin{array}{c}\text { Pasado } \\
\text { negativo }\end{array}$ & Futuro \\
\hline $\begin{array}{l}\text { Moral-Social- } \\
\text { Familiar }\end{array}$ & $\begin{array}{l}\text { C. de } \\
\text { correlación }\end{array}$ & $.05^{* * *}$ & .093 & -.076 & $.191^{*}$ \\
\hline $\begin{array}{l}\text { Afectivo- } \\
\text { Interpersonal }\end{array}$ & $\begin{array}{l}\text { C. de } \\
\text { correlación }\end{array}$ & $.380^{* * *}$ & -.065 & -.086 & $.237^{*}$ \\
\hline $\begin{array}{l}\text { Logros Materiales y } \\
\text { Prestigio }\end{array}$ & $\begin{array}{l}\text { C. de } \\
\text { correlación }\end{array}$ & $.315^{* * *}$ & $-.316^{* *}$ & -.174 & $.361^{* * *}$ \\
\hline $\begin{array}{l}\text { Logro Económico } \\
\text { Laboral }\end{array}$ & $\begin{array}{l}\text { C. de } \\
\text { correlación }\end{array}$ & $.312^{* * *}$ & -.097 & -.080 & $.213^{*}$ \\
\hline Afiliativa & $\begin{array}{l}\text { C. de } \\
\text { correlación }\end{array}$ & $.359^{* * *}$ & .144 & .074 & $.189^{*}$ \\
\hline Idealista-Altruista & $\begin{array}{l}\text { C. de } \\
\text { correlación }\end{array}$ & $.260^{* * *}$ & -.089 & -.018 & $.421^{* * *}$ \\
\hline Presión contextual & $\begin{array}{l}\text { C. de } \\
\text { correlación }\end{array}$ & $.215^{*}$ & $.262^{* * *}$ & .110 & -.076 \\
\hline $\begin{array}{l}\text { Capacitación como } \\
\text { Medio }\end{array}$ & $\begin{array}{l}\text { C. de } \\
\text { correlación }\end{array}$ & $.253^{* * *}$ & .092 & .106 & .087 \\
\hline Hedonista & $\begin{array}{l}\text { C. de } \\
\text { correlación }\end{array}$ & $.357^{* * *}$ & -.068 & .042 & .077 \\
\hline Estándar-Conformista & $\begin{array}{l}\text { C. de } \\
\text { correlación }\end{array}$ & .122 & $.210^{*}$ & .075 & .180 \\
\hline
\end{tabular}

Nota: C.: Coeficiente. Prueba Rho de Spearman. ${ }^{* *} p<.01 ;{ }^{*} p<.05$

Se encontraron las siguientes correlaciones significativas: El Presente Hedonista se relaciona de forma directa y moderada con la dimensión Moral-Social-Familiar, de manera directa pero débil con las dimensiones Afectivo-Interpersonal, Logros Materiales y de Prestigio, Logro Económico-Laboral, Afiliativa, Idealista-Altruista, Presión Contextual, Capacitación como Medio, Hedonista. El Presente Fatalista se relacionó de forma directa y débil con la Presión Contextual y con la Estándar- Conformista, de forma indirecta y débil con la meta Logros Materiales y Prestigio. Por último, el Futuro se relacionó de manera directa y débil con las metas de vida MoralSocial-Familiar, Afectivo-Interpersonal, Logros Materiales y de Prestigio, Logro Económico-Laboral, Afiliativa y se relaciona de manera directa pero moderada con la meta Idealista-Altruista. 


\section{Comparación de grupos}

En la comparación con la edad se utilizó la prueba U de Mann Whitney y se obtuvieron diferencias significativas en la dimensión Presente Fatalista y en dimensión presente Hedonista de la variable PTF (Tabla 2).

Tabla 2. Diferencias en la Perspectiva Temporal Futura en función de la Edad

\begin{tabular}{llcc}
\hline & & Rango Promedio & Sig. Asintótica \\
\hline Presente Hedonista & de 16 años o menos & 63.5 & $\mathbf{0 . 0 1 4}$ \\
& de 16 años o más & 48.3 & \\
\hline Presente Fatalista & de 16 años o menos & 64.71 & $\mathbf{0 . 0 0 3}$ \\
& de 16 años o más & 46.94 & 0.165 \\
\hline Pasado Negativo & de 16 años o menos & 59.39 & \\
& de 16 años o más & 50.99 & 0.289 \\
\hline Futuro & de 16 años o menos & 58.84 & \\
& de 16 años o más & 52.39 & \\
\hline
\end{tabular}

Tabla 3. Diferencias en las Metas de Vida en función de la Edad

\begin{tabular}{llcc}
\hline & & Rango Promedio & Sig. Asintótica \\
\hline Moral-Social-familiar & de 16 años o menos & 60.03 & .150 \\
& de 17 años o mas & 51.29 & \\
\hline Afectivo-Interpersonal & de 16 años o menos & 61.97 & .025 \\
& de 17 años o mas & 48.40 & \\
\hline Logros Materiales y & de 16 años o menos & 52.44 & .419 \\
Prestigio & de 17 años o mas & 57.33 & \\
\hline Logro Económico & de 16 años o menos & 56.86 & .666 \\
Laboral & de 17 años o mas & 54.24 & \\
\hline Afiliativa & de 16 años o menos & 58.50 & .340 \\
& de 17 años o mas & 52.71 & .256 \\
\hline Idealista-Altruista & de 16 años o menos & 59.08 & .181 \\
& de 17 años o mas & 52.18 & \\
\hline Presión Contextual & de 16 años o menos & 59.23 & .490 \\
& de 17 años o mas & 51.14 & \\
\hline Capacitación como & de 16 años o menos & 57.66 & .744 \\
Medio & de 17 años o más & 53.49 & .511 \\
\hline Hedonista & de 16 años o menos & 54.48 & \\
& de 17 años o más & 56.45 & \\
\hline Estándar-Conformista & de 16 años o menos & 57.56 & \\
& de 17 años o mas & 53.59 & \\
& &
\end{tabular}

Nota: Sig.: Significación. Prueba U de Mann-Whitney

Para comparar la orientación educativa se utilizó la prueba Kruskal-Wallis y se obtuvieron diferencias significativas en las dimensiones Metas Logro Económico laboral, Afiliativa y de Presión Contextual, de la variable metas de vida (Tabla 4). 
Tabla 4. Diferencias en las Metas de Vida en función de la Orientación educativa

\begin{tabular}{|c|c|c|c|}
\hline & & Rango Promedio & Sig. Asintótica. \\
\hline \multirow[t]{4}{*}{ Moral-Social-familiar } & Economía y Gestión & 45.96 & .237 \\
\hline & Ciencias Naturales & 61.12 & \\
\hline & Electromecánica & 61.15 & \\
\hline & Construcción & 52.33 & \\
\hline \multirow[t]{4}{*}{ Afectivo-Interpersonal } & Economía y Gestión & 49.54 & .164 \\
\hline & Ciencias Naturales & 57.93 & \\
\hline & Electromecánica & 65.46 & \\
\hline & Construcción & 47.60 & \\
\hline Logros materiales y & Economía y Gestión & 58.35 & .891 \\
\hline \multirow[t]{3}{*}{ Prestigio } & Ciencias Naturales & 56.17 & \\
\hline & Electromecánica & 53.63 & \\
\hline & Construcción & 51.98 & \\
\hline \multirow{4}{*}{$\begin{array}{l}\text { Logro Económico } \\
\text { Laboral }\end{array}$} & Economía y & 47.83 & .011 \\
\hline & Ciencias Naturales & 56.99 & \\
\hline & Electromecánica & 73.20 & \\
\hline & Construcción & 46.07 & \\
\hline \multirow[t]{4}{*}{ Afiliativa } & $\begin{array}{l}\text { Economía y } \\
\text { Gestión }\end{array}$ & 54.25 & .050 \\
\hline & Ciencias Naturales & 53.54 & \\
\hline & Electromecánica & 70.89 & \\
\hline & Construcción & 46.62 & \\
\hline \multirow[t]{4}{*}{ Idealista-Altruista } & Economía y Gestión & 60.69 & .068 \\
\hline & Ciencias Naturales & 64.01 & \\
\hline & Electromecánica & 51.93 & \\
\hline & Construcción & 44.05 & \\
\hline \multirow[t]{4}{*}{ Presión contextual } & $\begin{array}{l}\text { Economía y } \\
\text { Gestión }\end{array}$ & 44.63 & .007 \\
\hline & Ciencias Naturales & 55.75 & \\
\hline & Electromecánica & 74.16 & \\
\hline & Construcción & 48.17 & \\
\hline Capacitación como & Economía y Gestión & 54.50 & .952 \\
\hline \multirow[t]{3}{*}{ Medio } & Ciencias Naturales & 57.44 & \\
\hline & Electromecánica & 56.63 & \\
\hline & Construcción & 53.16 & \\
\hline \multirow[t]{4}{*}{ Hedonista } & Economía y Gestión & 55.54 & .941 \\
\hline & Ciencias Naturales & 56.06 & \\
\hline & Electromecánica & 58.13 & \\
\hline & Construcción & 52.72 & \\
\hline \multirow[t]{4}{*}{ Estándar-Conformista } & Economía y Gestión & 43.88 & .223 \\
\hline & Ciencias Naturales & 60.79 & \\
\hline & Electromecánica & 56.76 & \\
\hline & Construcción & 57.91 & \\
\hline
\end{tabular}

Nota: Sig.: Significación. Prueba U de Mann-Whitney 
A su vez se halló diferencias significativas en la dimensión Presente Hedonista de la variable PTF (Tabla 5).

Tabla 5. Diferencias en la Perspectiva Temporal Futura en función de la Orientación Educativa

\begin{tabular}{llcc}
\hline & & $\begin{array}{c}\text { Rango } \\
\text { Promedio }\end{array}$ & Sig. Asintótica \\
\hline Presente Hedonista & Economía y Gestión & $\mathbf{5 0 , 0 4}$ & $\mathbf{. 0 0 1}$ \\
& Ciencias Naturales & $\mathbf{6 8 , 3 2}$ & \\
& Electromecánica & $\mathbf{6 5 , 0 2}$ & \\
& Construcción & $\mathbf{3 7 , 4 2}$ & .144 \\
\hline Presente Fatalista & Economía y Gestión & 45,5 & \\
& Ciencias Naturales & 60,47 & \\
& Electromecánica & 64,07 & .151 \\
& Construcción & 51,16 & \\
\hline Pasado Negativo & Economía y Gestión & 52,52 & .352 \\
& Ciencias Naturales & 64,93 & \\
& Electromecánica & 52,07 & \\
& Construcción & 47,48 & \\
\hline Futuro & Economía y Gestión & 53,98 & \\
& Ciencias Naturales & 62,46 & \\
& Electromecánica & 56,17 & \\
& Construcción & 48,07 & \\
\hline
\end{tabular}

Nota: Sig.: Significación. Prueba U de Mann-Whitney

\section{CONCLUSIÓN}

Se planteó como objetivo principal el análisis y la relación entre las dimensiones de la variable PTF y Metas de Vida en adolescentes estudiantes de nivel medio. Los resultados indican que en los adolescentes con orientación al presente hedonista prevalece más el deseo que las metas concretas, en el presente fatalista los adolescentes tienden a conformarse mientras que en el pasado negativo esto no sucedería, además capacitarse para los adolescentes no constituiría un medio para alcanzar las metas establecidas.

Más específicamente, con respecto a la primera hipótesis de trabajo, los resultados obtenidos confirman la misma. Esto indicaría que las personas que viven el presente realizan solo aquello que tienen ganas posponiendo las responsabilidades el mayor tiempo posible. Los proyectos estarían apuntados a la satisfacción personal que puedan obtener de los mismos. Estos resultados son congruentes con lo planteado por Zimbardo \& Boyd (1999) que indican que los sujetos en los que prevalece la orientación al presente hedonista tienden a actuar de acuerdo con consecuencias que son principalmente inmediatas, limitándose a disfrutar del momento, con objetivos y concretos apuntados a la gratificación personal. A su vez, Cattaneo \& Schmidt (2014) plantean que este tipo de metas son falsas salidas, que no presentan un proyecto real porque lo que estaría imperando en estos adolescentes seria la inmediatez y la fantasía (por ejemplo; con mi futura profesión quiero servir a la comunidad y esto sería producto de las tramitaciones psíquicas propias del desarrollo.

En relación con la segunda hipótesis, los resultados obtenidos confirman de manera parcial la misma, los adolescentes que tienen una actitud negativa hacia el presente, caracterizada por una sensación de indefensión 0 resignación, aceptan y se conforman ante las dificultades que el contexto presenta, planificando actividades en función de la presión o mandatos que el contexto familiar y social exige, pero esto no sucedería con aquellos han tenido experiencias difíciles y complicadas en el pasado. A su vez, en ambas orientaciones temporales se evidencia la ausencia de metas concretas y planificadas con respecto al futuro. Por un lado, estos resultados concuerdan con lo que plantean Zimbardo \& Boyd (1999) ya que sostienen que las valoraciones, experiencias y creencias que el sujeto tiene sobre sí mismo y su presente ejerce un papel decisivo en la planificación y toma de decisiones con 
respecto al futuro, y si estas son negativas aumentará la posibilidad de conformarse frente a las dificultades que plantee el contexto (Cattaneo \& Schmidt, 2014). Por otro lado, a partir de los resultados hallados con respecto al pasado negativo surgió la pregunta acerca de que las personas que hayan tenido dificultades o malas experiencias en su pasado presenten algún tipo de motivación y se revelen frente a las posibilidades y exigencias que el contexto presenta en pos de evitar que se repitan sucesos del pasado o quizá reivindicarse en el futuro, considerando que esta etapa, caracterizada por un proceso de transformación sería propicia para revertir la visión negativa del pasado y encaminarse hacia la autorrealización que según Cattaneo y Schmidt (2014) constituyen la finalidad de la existencia humana. Por último, la investigación llevada a cabo por Di Fabio de Anglat, Noriega \& Vásquez (2018) evidenciaron que la orientación al pasado y presente negativo se asocia al establecimiento de metas relacionadas a logros académicos, laborales y relacionados al self, sin embargo, esto no se evidenció en la presente investigación.

Con relación a la tercera hipótesis, los resultados obtenidos, indican que la misma se confirma de manera parcial. Esto indica que las metas se formulan en función de lograr liderazgo y lucimiento personal, de obtener una recompensa económica a partir del propio esfuerzo 0 de la satisfacción personal que se pudiera obtener del cumplimiento de estas, sin embargo, estudiar o formarse para los adolescentes no constituye un medio para lograr cierta meta establecida. Las investigaciones realizadas sobre la PTF sostienen que la orientación al Futuro se asocia a aspectos positivos que permiten desarrollar mejores estrategias de planificación, alcanzar logros laborales, académicos y materiales entre otros (Lens, Paixaó, Herrera \& Grobler, 2012; Fernández \& Macbeth, 2018). Los adolescentes que formaron parte de la muestra pertenecen a la denominada generación Z o Centennial que se caracteriza por haber nacido en un contexto social con medios tecnológicos y de comunicación a su alcance, los cuales utilizan como un recurso en forma productiva y los consideran parte de la vida cotidiana (Cuesta et al., 2009) es por esto que, es posible pensar que capacitarse para esta nueva generación no es el único camino posible para conseguir un trabajo bien remunerado, a diferencia de generaciones anteriores que veían el estudio 0 formación como única posibilidad para lograr el éxito y ser alguien en la vida.

La cuarta hipótesis se refuta ya que se han encontrado diferencias en las metas Afectivo-Interpersonal. Esto indica que en los adolescentes de menor edad habría mayor presencia de metas apuntadas a lograr una mayor estabilidad emocional, familiar o social que en los adolescentes que tienen mayor edad. Hasta el momento no se ha encontrado en estudios anteriores comparaciones en este rango etario, por lo tanto, no hay antecedentes que permitan discutir los resultados hallados.

La quinta hipótesis se confirma esto indicaría que los adolescentes de menor edad se orientarían a disfrutar y vivir del momento presente sin planificar demasiado su futuro sobre el cual, a su vez, presentarían una actitud de falta de esperanza y de resignación mayor que los adolescentes de más edad. Este resultado concuerda con la investigación realizada por Galarraga y Stover (2017) quienes hallaron que los adolescentes entre 14 y 15 se orientan al presente fatalista y con los aportes de diversos autores (Carcelerén Velarde \& Martínez Uribe, 2008; Diaz Morales, 2006) que también hallaron un predominio del presente hedonista en los de menor edad.

La sexta hipótesis se confirma. Esto indicaría que los estudiantes con orientación Electromecánica apuntan sus objetivos a obtener un trabajo bien remunerado a partir del título obtenido, pero esto no sucedería con los estudiantes de Construcciones. Este resultado podría interpretarse en función de la salida laboral estiman tendrán los egresados de las escuelas técnicas. Los egresados con título "Técnico en electromecánica" poseen un campo de trabajo mucho más amplio que el de los egresados con título "Maestro mayor de obras" es por lo que, estimarían conseguir empleo más rápidamente.

A modo de cierre, es conveniente considerar las limitaciones que presenta este trabajo relacionadas en primer lugar al apareamiento de la muestra en cuestiones como el género y en segundo lugar con el estado del arte ya que en la búsqueda bibliográfica tanto en Argentina como en otros países no se encontraron correlaciones entre las variables trabajadas utilizando el instrumento Metas de Vida desarrollado por Cattaneo y Schmidt (2014), y escasas comparaciones relacionadas con la franja etaria trabajada. 
Finalmente, en próximas investigaciones se podría indagar sobre las metas a corto y largo plazo que presentan los adolescentes y de esta manera obtener más información sobre el vínculo de estas con la orientación temporal, pudiendo así obtener un mayor conocimiento del tema que permita desarrollar programas orientados a estimular a los adolescentes a pensar en su futuro.

\section{REFERENCIAS BIBLIOGRÁFICAS}

Austin, J., \& Vancouver, J. (1996). Construcciones de objetivos en psicología: estructura, proceso y contenido. Boletín psicológico, 120(3), 338-375.

Carcelén Velarde, M., \& Martínez, U. (2008). Perspectiva temporal futura en adolescentes institucionalizados. Revista de Psicología, 26(2), 255-278.

Cattaneo, M., \& Schmidt, V. (2014). Escala de Metas de Vida. Editorial Paidós.

Cuesta, E., Ibáñez, M., Tagliabue, R., \& Zangaro, M. (2009) El cerebro de los "millennials" está hecho de otra pasta y por eso responde de manera diferente a los medios digitales.

Daura, F., \& Durand, J. (2018). ¿Cuán involucrado estoy? Un estudio exploratorio sobre el compromiso académico y la orientación temporal en estudiantes argentinos. Revista Panamericana de Pedagogía, 26, 73-95.

Del Río, M., \& Herrera, A. (2006). Desarrollo de un instrumento para evaluar perspectiva de tiempo futuro en adolescentes. Avances en Medición, 4, 47-60.

Díaz Morales, J., \& Martínez Patricia (2004). Metas y satisfacción vital en adolescentes españoles y peruanos según género y nivel socioeconómico. Revista de Psicología de la PUCP, 22(19).

Difabio de Anglat, H., Vásquez, S., \& Noriega Biggio, M. (2018). Orientación temporal y metas vitales en estudiantes argentinos. Revista de Psicología, 36(2).

Fernández, H., \& Macbeth, G. (2018). Perspectiva de tiempo futuro, metas y sub-metas: su rol en la toma de decisiones. Revista Latinoamericana de Ciencia psicológica, 10(2).

Ferrari, J. \& Díaz-Morales, J. (2007). Dilatación: diferentes orientaciones del tiempo reflejan diferentes motivos. _. Journal of Research in Personality, 41(3), 707-714.

Ford, M. (1992). Motivating Humans. Goals, emotions, and personal agency beliefs. Nebury Park: C.A: Sage.

Fraisse, P. (1983). Le future dans les perspective temporelles. Intenational Journal of Pychology, 18, 489-492.

Galarraga, M., \& Stover, J. (2016). Inventario de Perspectiva temporal de Zimbardo: Adaptación en estudiantes de nivel medio de Buenos Aires. Psicodebate, 16(1), 109-128.

Galarraga, M., \& Stover, J. (2017). Perspectiva Temporal Futura y estrategias de afrontamiento en estudiantes de nivel medio próximos a egresar. Investigaciones en Psicología, 22(2), 37-45.

Giuliani, M., Arias, C., \& Villar Posada, F. (2014). Diferencias según grupo de edad de las características de la percepción temporal futura, las metas vitales y las estrategias de regulación de la ira y la tristeza en escenarios familiares. Anuario de Proyectos e Informes de Becarios de Investigación, 11.

Huertas, J (2003). Motivación: Queres aprender. Buenos Aires: Aique.

Kasser, T. (2014) Commissioned paper: values and human wellbeing. Galesburg, Il: Knox College.

Lenning, C., Burns A., \& Conney, G. (1998). Profiles of time perspective and personality: developmental considerations. The Journal of Psychology, 132, 629-641.

Lens, W., Paixaó, M., Herrara, D., \& Grobler, A. (2012). La perspectiva del Tiempo Futuro como variable motivacional: el contenido y la extensión de los objetivos futuros afectan la cantidad y la calidad de la motivación. Japanese Pychological Reseach, 54(3).

Lewin, K. (1935). A dynamic Theory of Personality. New York: McGrawHill Book Co.

Lewin, K. (1942). Time perspective and morale. En G. Watson (Ed.). Civilian Morales. Boston: Houghton Mifflin.

Lilo Espinosa, J. (2004). Crecimiento y comportamiento en la Adolescencia. Revista de la Asociación Española de Neuropsiquiatría, 90, 57-71.

Martínez, K. (2003). Desarrollo y evaluación de un programa de intervención breve para adolescentes que inician el consumo de alcohol y drogas. Tesis doctoral. México. 
Robles Ojeda, F., Xochitl Galicia Moyeda, I., \& Sánchez Velasco, A. (2017). Orientación Temporal, autorregulación y aproximación al aprendizaje en el rendimiento académico en estudiantes universitarios. Revista Electrónica de Psicología Iztacala 20(2).

Wentzel, K. (1992). Motivation and achievement in adolescence. A multiple goal perspective. En Shunck, D. \& Meence, J (Eds.) Students perceptions in the classroom: Causes and consequences, 287-306, Hillsdale. N.J: Erlbaum.

Zimbardo, P., \& Boyd, J. (1999). Poner el tiempo en perspectiva: una métrica de diferencias individuales válida y confiable. Revista de Personalidad y Psicología Social, 77(6), 1271-1288. 